\title{
Successful treatment of multidrug resistant Acinetobacter baumannii meningitis
}

\author{
Suzan Saçar, ${ }^{1}$ H. Turgut, ${ }^{1}$ D. Hırçın Cenger, ${ }^{1}$ E. Coşkun, ${ }^{2}$ A. Asan,,${ }^{1}$ I. Kaleli. ${ }^{3}$ \\ ${ }^{1}$ Pamukkale University Hospital, Infectious Diseases and Clinical Microbiology Department; ${ }^{2}$ Pamukkale University Hospital, \\ Neurosurgery Department; ${ }^{3}$ Pamukkale University Hospital, Microbiology and Clinical Microbiology Department; Denizli, Turkey.
}

\begin{abstract}
Background: Acinetobacter baumannii is a major cause of nosocomial infections in many hospitals and appears to have a propensity for developing multiple antimicrobial resistance rapidly.

Cases: We report two cases with post-surgical meningitis due to multidrug resistant $A$. baumannii which were successfully treated with high-dose intravenous meropenem therapy.

Conclusions: Multidrug resistant Acinetobacter spp. in intensive care units are a growing concern. High-dose meropenem is used in the treatment of these infections.
\end{abstract}

Key Words: Acinetobacter baumannii, Post-surgical meningitis, High dose meropenem.

J Infect Developing Countries 2007; 1(3):342-344.

Received 9 August 2007 - Accepted 15 November 2007.

Copyright (C) 2007 Saçar et al. This is an open access article distributed under the Creative Commons Attribution License, which permits unrestricted use, distribution, and reproduction in any medium, provided the original work is properly cited.

\section{Introduction}

A. baumannii is a major cause of nosocomial infections in worldwide. The infection caused by Acinetobacter is difficult to control due to multidrug resistance, which limits therapeutic options in critically ill and debilitated patients, especially those in intensive care units [1]. We report two cases of post-surgical meningitis due to multidrug resistant $A$. baumannii occurring in patients admitted in the same neurosurgical intensive care unit of Pamukkale University Hospital, Turkey. Upon institution of therapy with high doses of meropenem, the patients experienced successful clinical and microbiological outcomes.

\section{Case 1}

A 52-year-old man developed meningitis due to $A$. baumannii 4 days after placement of a ventriculostomy catheter for management of post traumatic left temporal lobe hemorrhage. Prior to the development of meningitis, the hospital course was significant for ventilator-associated pneumonia and receipt of multiple courses of broad-spectrum antibiotics. Seven days later, cerebrospinal fluid (CSF) was turbid and its examination revealed neutrophil pleocytosis. $A$. baumannii was isolated from the culture of the CSF. The following antimicrobial agents were tested and the microorganism was resistant to ampicillin, amoxycillin/clavulanic acid, cephalothin, ceftriaxone, cefixime, ceftazidime, cefuroxime, cephotaxime, ciprofloxacin, ofloxacin, fosfomycin, trimethoprim-sulphamethoxazole, aztreonam, tetracycline, piperacillin-tazobactam, piperacillin, amikacin, tobramycin, and gentamicin except carbapenems. The therapy with intravenous meropenem (daily total dose 6 grams) was started and the extraventricular drainage system was removed. CSF cultures became sterile at the forth day of the treatment. High-dose intravenous meropenem therapy continued 21 days. Laboratory results of CSF are shown in Table 1.

\section{Case 2}

A 62-year-old woman was admitted to Pamukkale University Hospital because of cerebellar hematoma and aspiration pneumonia. A ventriculostomy catheter was used on the patient for management of cerebellar hematoma. Post-operatively, her fever persisted and she developed convulsions and deterioration in the level of consciousness. The hospital course was 
significant for aspiration pneumonia, bloodstream infection, and receipt of multiple courses of broadspectrum antibiotics. Multidrug resistant $A$. baumannii was isolated from the CSF. The following antimicrobial agents were tested and the microorganism was resistant to ampicillin, amoxycillin/clavulanic acid, cephalothin, ceftriaxone, cefixime, ceftazidime, cefuroxime, cephotaxime, ciprofloxacin, ofloxacin, fosfomycin, trimethoprim-sulphamethoxazole, aztreonam, tetracycline, piperacillin-tazobactam, piperacillin, amikacin, tobramycin, and gentamicin except carbapenems. Diagnosis was accepted as hospital acquired meningitis due to $A$. baumannii. The therapy with intravenous meropenem (daily total dose 6 grams) was started and continued 21 days. The ventriculostomy catheter was removed on the 32nd day of her hospitalization and the CSF remained sterile from $A$. baumannii throughout the rest of the hospitalization. Laboratory results of CSF are shown in Table 1.

Table 1. Laboratory results of CSF.

\begin{tabular}{|c|c|c|c|c|c|c|c|}
\hline \multirow{2}{*}{$\begin{array}{l}\text { Day of } \\
\text { treatment }\end{array}$} & \multicolumn{2}{|c|}{$\begin{array}{c}\text { CSF } \\
\text { [cell/mm] }\end{array}$} & \multicolumn{2}{|c|}{ Type of CSF cells } & \multicolumn{2}{|c|}{ Microorganism } & \multirow{2}{*}{$\begin{array}{c}\text { Meropenem } \\
\text { Treatment } \\
\begin{array}{c}\text { Cases } 1 \\
\text { and 2 }\end{array} \\
\end{array}$} \\
\hline & $\begin{array}{c}\text { Case } \\
1\end{array}$ & $\begin{array}{c}\text { Case } \\
2\end{array}$ & Case 1 & Case 2 & Case 1 & Case 2 & \\
\hline $1^{\circ}$ day & 1940 & - & PMNL* & $\begin{array}{l}\text { PMNL+ } \\
\text { erythrocyte }\end{array}$ & A.baumannii & A.baumannii & 6 gr/day \\
\hline $3^{\circ}$ day & 2000 & - & PMNL & $\begin{array}{l}\text { PMNL+ } \\
\text { erythrocyte }\end{array}$ & A.baumannii & A.baumannii & $6 \mathrm{gr} / \mathrm{day}$ \\
\hline $4^{\circ}$ day & 90 & - & PMNL & $\begin{array}{l}\text { PMNL+ } \\
\text { erythrocyte }\end{array}$ & - & A.baumannii & $6 \mathrm{gr} / \mathrm{day}$ \\
\hline $5^{\circ}$ day & 450 & - & $\begin{array}{c}\text { PMNL+ } \\
\text { Lymphocyte }\end{array}$ & $\begin{array}{l}\text { PMNL+ } \\
\text { erythrocyte }\end{array}$ & - & A.baumannii & $6 \mathrm{gr} / \mathrm{day}$ \\
\hline $6^{\circ}$ day & 100 & - & $\begin{array}{l}\text { PMNL+ } \\
\text { Lymphocyte }\end{array}$ & - & - & A.baumannii & $6 \mathrm{gr} / \mathrm{day}$ \\
\hline $7^{\circ}$ day & 250 & - & $\begin{array}{l}\text { PMNL+ } \\
\text { Lymphocyte }\end{array}$ & - & - & A.baumannii & 6 gr/day \\
\hline $10^{\circ}$ day & 10 & 30 & $\begin{array}{c}\text { PMNL+ } \\
\text { Lymphocyte }\end{array}$ & - & - & A.baumannii & $6 \mathrm{gr} / \mathrm{day}$ \\
\hline $14^{\circ}$ day & 0 & - & - & - & - & - & $6 \mathrm{gr} / \mathrm{day}$ \\
\hline
\end{tabular}

\section{Discussion}

Acinetobacter species are aerobic gram negative coccobacilli that have emerged as important opportunistic pathogens, especially among debilitated patients. A. baumannii can be a causal agent of diseases such as pneumonia, bacteremia, meningitis, soft tissue and urinary tract infections associated with high mortality [2]. Jover Saenz et al. [3] reported that the overall annual incidence of nosocomial infections by multiresistant pathogens was 4.0 per 1,000 patients admitted. $A$. baumannii showed the highest incidence, particularly in the intensive care units (15.4 per 1,000 patients admitted).

A. baumannii is a bacterium that appears to have a propensity for developing multiple antimicrobial resistance [1]. Because of frequent resistance to aminoglycosides, fluoroquinolones, ureidopenicillins and third-generation cephalosporins, carbapenems are important agents for managing Acinetobacter infections. Carbapenem resistance has been reported with many outbreaks of Acinetobacter spp. Many of these strains remain susceptible only to polymyxin, a toxic peptide antibiotic. The rapid evolution of drug resistance has severely limited the options for effective therapy for infections caused by this pathogen [2]. The mortality rate of patients with $A$. baumannii was significantly higher than that of patients without infection [4]. Vivek et al. [2] found that Acinetobacter species accounted for $10 \%$ of Gram negative isolates and from these isolates only half of Acinetobacter species were susceptible to carbapenems; 11 hospitals had at least one isolate resistant to carbapenems. Other Acinetobacter susceptibility rates were as follows: polymyxin, amikacin, ampicillin/sulbactam, ceftazidime and ciprofloxacin. Yaman et al. [5] reported that meropenem was the most effective antibiotic against gram negative organisms (89\%); this was followed by imipenem (87.2\%) and piperacillin/tazobactam.

In many adults with acute bacterial meningitis, Acinetobacter spp. were found to be responsible for approximately $10 \%$ of Gram negative bacillary and $4 \%$ of all nosocomial meningitis [6]. Postneurosurgical meningitis is a serious complication. When this disease is caused by a multidrugresistant $A$. baumannii, the management of such a case is challenging. When the infection involves the meninges, the choice of an antibiotic is further limited by the blood-brain barrier. There are differences between the CSF concentrations of meropenem and imipenem. The diffusion of both drugs into the healthy meninges is very low (1$10 \%)$; however, when the meninges become inflamed, the diffusion increases. For imipenem this rate is about $15 \%$, but for meropenem it is increased up to 45-50\%. Adverse effects, especially neurotoxicity, are lower in meropenem usage than in imipenem. For these reasons meropenem treatment is suggested for cases of meningitis. The recommended dosage is 6 grams per day [7]. In our cases, meropenem therapy was given due to $A$. baumannii meningitis and there was a clinical and laboratory response in a few days. In our cases CSF remained free of $A$. baumannii throughout the rest of the 
hospitalization. Our case reports are limited in that we were unable to conduct molecular analysis so we did not determine whether the strains were the same or not.

In conclusion, in the last two decades Acinetobacter baumannii has emerged as a major relevant world nosocomial pathogen. Postneurosurgical meningitis is a serious complication when multidrug resistant $A$. baumannii are isolated. In our cases we presented the potential utility of high-dose meropenem for meningitis due to resistant $A$. baumannii. New antibiotics and new therapeutic strategies are needed to combat this multidrug resistant pathogen.

\section{References}

1. Cisneros JM, Rodríguez-Baño J (2002) Nosocomial bacteremia due to Acinetobacter baumannii: epidemiology, clinical features and treatment. Clin Microbiol Infect 8: 687-693.

2. Manikal VM et al. (2000) Endemic Carbapenem-Resistant Acinetobacter Species in Brooklyn, New York: Citywide Prevalence, Interinstitutional Spread, and Relation to Antibiotic Usage. Clinical Infectious Diseases 31: 101106.
3. Jover Saenz A et al. (2005) Nosocomial infection by multiresistant pathogens during one year in a secondary hospital: clinical and epidemiological analysis. An Med Interna. 22: 59-64.

4. Theaker C, Azadian B, Soni N (2003) The impact of Acinetobacter baumannii in the intensive care unit. Anaesthesia 58: 271-274.

5. Yaman A et al. (2004) Investigation of the antibiotic susceptibility patterns of pathogens causing nosocomial infections. Saudi Med J. 25: 1403-1409.

6. Durand ML et al. (1993) Acute bacterial meningitis in adults. A review of 493 episodes. N Engl J Med 328: 2128.

7. Çakır N (2003) Carbapenems, Leblebicioğlu H, Usluer G, Ulusoy S, editors. Antibiotics. Ankara: Bilimsel Tıp Yayınevi Press. 275-286.

Corresponding Author: Suzan Saçar, Pamukkale University Faculty of Medicine, Infectious Diseases and Clinical Microbiology Department, Kınıklı 20070 Denizli, Turkey, Tel: +90 02582118585 / 2293, Fax: 2134922, e-mail: suzansacar@yahoo.com

Conflict of interests: The authors declare that they have no conflict of interests. 